\title{
Baicalin inhibits PDGF-BB-stimulated vascular smooth muscle cell proliferation through suppressing PDGFRß-ERK signaling and increase in p27 accumulation and prevents injury-induced neointimal hyperplasia
}

\author{
Li-Hua Dong ${ }^{1}$, Jin-Kun Wen ${ }^{1}$, Sui-Bing Miao ${ }^{1}$, Zhenhua Jia ${ }^{2}$, Hai-Juan $\mathrm{Hu}^{1}$, Rong-Hua Sun ${ }^{1}$, Yiling Wu ${ }^{2}$, \\ Mei $\operatorname{Han}^{1}$ \\ ${ }^{I}$ Department of Biochemistry and Molecular Biology, Institute of Basic Medicine, Key Laboratory of Neural and Vascular Biol- \\ ogy, China Ministry of Education, No. 361, Zhongshan East Road, Shijiazhuang 050017, China; ${ }^{2}$ Integration of Traditional and \\ Western Medical Research Academy of Hebei Province, No.238, Tianshan Street, Hebei Medical University, Shijiazhuang 050017, \\ China
}

The increased proliferation and migration of vascular smooth muscle cells (VSMCs) are key events in the development of atherosclerotic lesions. Baicalin, an herb-derived flavonoid compound, has been previously shown to induce apoptosis and growth inhibition in cancer cells through multiple pathways. However, the potential role of baicalin in regulation of VSMC proliferation and prevention of cardiovascular diseases remains unexplored. In this study, we show that pretreatment with baicalin has a dose-dependent inhibitory effect on PDGF-BB-stimulated VSMC proliferation, accompanied with the reduction of proliferating cell nuclear antigen (PCNA) expression. We also show that baicalin-induced growth inhibition is associated with a decrease in cyclin E-CDK2 activation and increase in p27 level in PDGF-stimulated VSMCs, which appears to be at least partly mediated by blockade of PDGF receptor $\beta$ (PDGFR $\beta$ )-extracellular signal-regulated kinase 1/2 (ERK1/2) signaling. In addition, baicalin was also found to inhibit adhesion molecule expression and cell migration induced by PDGF-BB in VSMCs. Furthermore, using an animal carotid arterial balloon-injury model, we found that baicalin significantly inhibited neointimal hyperplasia. Taken together, our results reveal a novel function of baicalin in inducing growth arrest of PDGF-stimulated VSMCs and suppressing neointimal hyperplasia after balloon injury, and suggest that the underlying mechanism involves the inhibition of cyclin E-CDK2 activation and the increase in p27 accumulation via blockade of the PDGFR 3 -ERK1/2 signaling cascade.

Keywords: baicalin; vascular smooth muscle cells; proliferation; cyclin E; neointimal hyperplasia

Cell Research (2010) 20:1252-1262. doi:10.1038/cr.2010.111; published online 27 July 2010

\section{Introduction}

The increased proliferation and migration of vascular smooth muscle cells (VSMCs) are key events in the

Correspondence: Mei Han ${ }^{\mathrm{a}}$, Yiling $\mathrm{Wu}^{\mathrm{b}}$

${ }^{\mathrm{a}}$ Tel: +86-0311-86265563; Fax: +86-0311-86266180

E-mail: hanmei@hebmu.edu.cn

${ }^{\mathrm{b}}$ Tel: +86-0311-85901553; Fax: +86-0311-85901088

E-mail: jiatcm@163.com

Received 26 January 2010; revised 16 May 2010; accepted 7 June 2010; published online 27 July 2010 development of atherosclerosis and restenosis. Both of these events can be induced by cytokines and growth factors, such as platelet-derived growth factor (PDGF) [1]. PDGF initiates a multitude of biological effects through the activation of intracellular signal transduction pathways that contribute to VSMC proliferation, migration, and collagen synthesis [2]. The cell cycle is a common convergent point for the mitogenic signaling cascades. Progression through several major checkpoints in the cell cycle is controlled by multiple protein kinases, each of which contains a regulatory cyclin component and a catalytic cyclin-dependent kinase (CDK) [3]. The expression 
<smiles>O=C1CC(c2ccccc2)Oc2cc(O)c(O)c(O)c21</smiles>

Baicalein

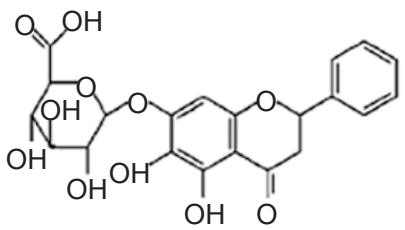

Baicalin
Figure 1 Chemical structure of baicalin and baicalein.

levels of each component, their phosphorylation status, and the presence of specific CDK inhibitors regulate the activity of these kinases. p2 $7^{\text {kip1 }}$ (p27) is a negative regulator of the protein kinase $\mathrm{CDK} 2 /$ cyclin $\mathrm{E}$, and can block

A

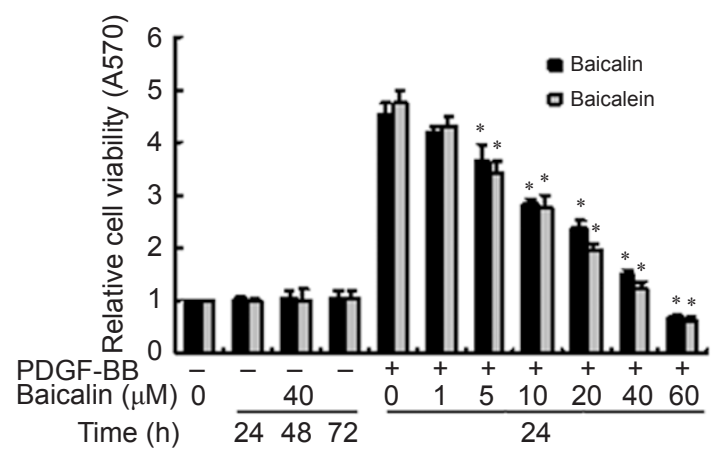

C

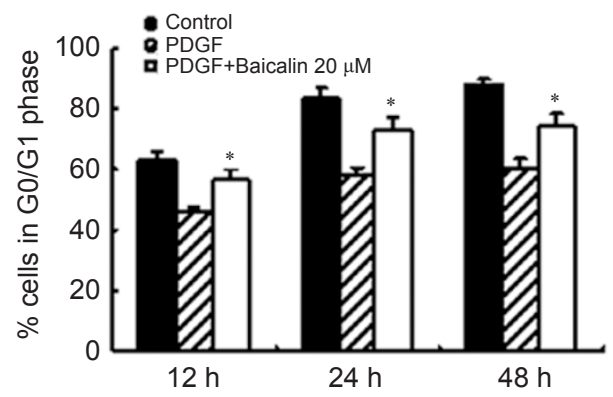

$E$

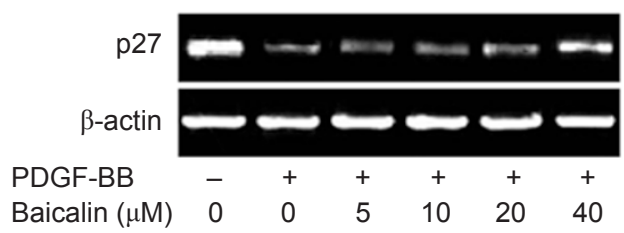

the cell cycle at $\mathrm{G} 0 / \mathrm{G} 1$ phase [4]. The levels of p27 are high in the G0/G1 phases of cell cycle. Upon mitogenic stimulation, p27 is rapidly degraded, thus allowing the action of $\mathrm{CDK} 2 /$ cyclin $\mathrm{E}$ to promote cell proliferation [5]. ERK1/2 signaling is necessary for the degradation or downregulation of p27, and is also crucial in mediating VSMC proliferation in response to a multitude of mitogenic stimuli and vascular injury $[6,7]$. Therefore, agents that can regulate the cell cycle processes in VSMCs may have a role in the prevention and treatment of atherosclerosis and restenosis after angioplasty.

Scutellaria baicalensis has been shown to harbor a broad spectrum of biological activities, including antiinflammatory, antioxidant and antiproliferation activities, based on its long history in clinical applications. Baic-

B

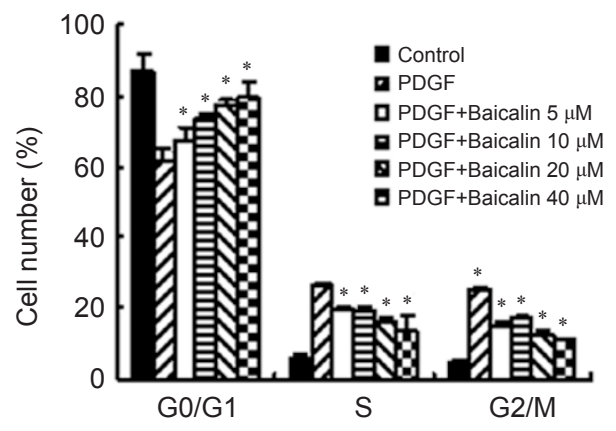

$\mathrm{D}$
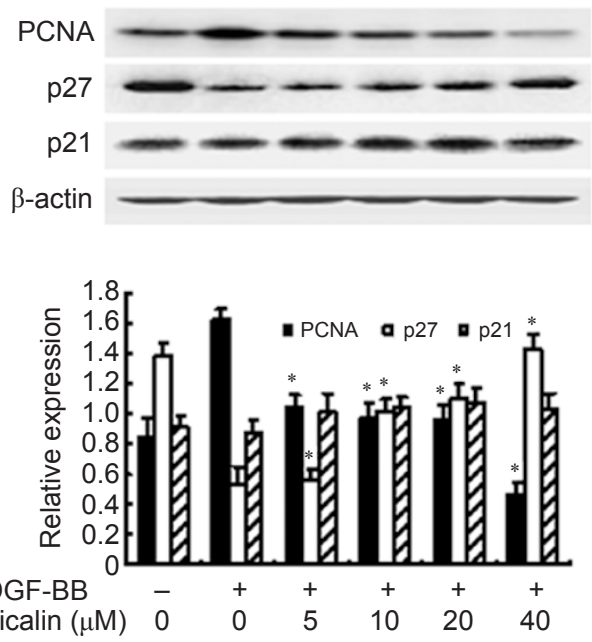

Figure 2 Baicalin inhibits proliferation of VSMCs induced by PDGF-BB. (A) MTT assays. VSMCs were pretreated by various concentrations of baicalin or baicalein for indicated time periods, and then stimulated with PDGF-BB for $24 \mathrm{~h}(n=5)$. (B, C) Flow cytometric analysis $(n=5)$. (D) Western blotting. The graphs represent the relative level of the proteins in four independent experiments. (E) RT-PCR. $\beta$-Actin was used as an internal control. VSMCs were pretreated by indicated concentrations of baicalin for $24 \mathrm{~h}$ followed by $24 \mathrm{~h}$ of PDGF-BB stimulation. (B-E) $* P<0.05$, compared with PDGF-BB. 
alin and baicalein are flavonoids derived from the root of Scutellaria baicalensis Georgi (Figure 1). It has been reported that baicalein, which has the same backbone structure as baicalin, shows a stronger cardioprotective effect and can inhibit the proliferation of cancer cells via induction of apoptosis [8-11]. A recent study showed that baicalin pretreatment reduced the hyperthermia, intracranial hypertension, and increase of nitric oxide metabolite levels during heatstroke; baicalin also suppressed the heatstroke-induced increase in IL- $1 \beta$ and TNF- $\alpha$ levels [12]. Baicalin may thus protect against cerebrovascular dysfunction and brain inflammation in heatstroke [13]. Although baicalin has been also found to induce apoptosis and to inhibit inflammation through multiple pathways, little is known about the role of baicalin in regulating VSMC proliferation and prevention of restenosis. In this study, we show that baicalin has an inhibitory effect on PDGF-BB-stimulated proliferation and migration of VSMCs, and prevents neointimal hyperplasia induced by balloon injury. We also show that baicalin-induced growth inhibition is associated with the inhibition of cyclin E-CDK2 activation and an increase of p27 levels in PDGF-stimulated VSMCs. This effect of baicalin is mediated, at least partly, through blockade of the PDGF receptor $\beta$ (PDGFR $\beta$ )-extracellular signal-regulated kinase $1 / 2($ ERK1/2) signaling cascade.

\section{Results}

Baicalin inhibits PDGF-BB-induced proliferation of VSMCS

Baicalin has been demonstrated as an active antitumor compound that leads to cell cycle arrest and growth suppression in cancer cells $[8,9]$. We first examined the effect of baicalin on PDGF-BB-induced proliferation of VSMCs using the MTT assay. VSMCs were pretreated with different concentrations $(1-60 \mu \mathrm{M})$ of baicalin followed by stimulation with PDGF-BB $(10 \mathrm{ng} / \mathrm{ml})$ for 24 h. Baicalin inhibited the PDGF-BB-induced proliferation of VSMCs in a concentration-dependent manner (Figure $2 \mathrm{~A}$ ). The IC50 value (concentration needed for $50 \%$ growth inhibition) of baicalin was about $16.39 \mu \mathrm{M}$ under the present experimental conditions. Higher concentrations of baicalin $(40$ and $60 \mu \mathrm{M})$ almost completely blocked the cell proliferation induced by PDGF-BB (Figure 2A). When quiescent cells were treated with baicalin $(40 \mu \mathrm{M})$ for $24-72 \mathrm{~h}$ in the absence of PDGF-BB, no significant difference was observed in the viability of VSMCs as compared with the untreated cells, suggesting that baicalin is not cytotoxic at the concentrations tested (Figure 2A). To determine the effect of baicalin on cell cycle progression, VSMCs were pretreated with 5-40 $\mu \mathrm{M}$ baicalin before PDGF-BB stimulation for $24 \mathrm{~h}$, and then subjected to flow cytometry analysis. Baicalin (10-40 $\mu \mathrm{M})$ significantly increased the fraction of G0-G1 phase cells, but decreased the numbers of G2-M and $\mathrm{S}$ phase cells in VSMCs $(P<0.05)$ (Figure 2B). Treatment with $20 \mu \mathrm{M}$ baicalin for $24 \mathrm{~h}$ increased the fraction of $\mathrm{G} 0$ G1 phase cells by $\sim 15 \%$ compared with PDGF-BB alone (Figure 2C). Inhibition of cell proliferation by baicalin was verified by its ability to reduce proliferating cell nuclear antigen (PCNA) expression and to elevate p27 levels in a concentration-dependent manner, coinciding with cell cycle arrest (Figure 2D). Treatment with $40 \mu \mathrm{M}$ baicalin resulted in an twofold increase in the p27 protein level relative to that of PDGF-BB alone, whereas a slight induction of $\mathrm{p} 27 \mathrm{mRNA}$ level by baicalin was also observed (Figure 2D and 2E). However, the expression of p21 protein was not markedly altered by baicalin. Together, these data show that baicalin inhibits PDGF-BBinduced proliferation of VSMCs, which is accompanied by increased $\mathrm{p} 27$ expression.

\section{Baicalin inhibits cyclin E-CDK2 activation and p27} phosphorylation in VSMCs

Cell cycle progression is controlled by cyclins and CDKs. To determine the expression of cyclins and CDKs in baicalin-treated VSMCs, the lysates of VSMCs were analyzed by western blotting. Treatment with baicalin (5-40 $\mu \mathrm{M})$ reduced PDGF-BB-induced expression of cyclin $\mathrm{E}$ and $\mathrm{CDK} 2$ in a concentration-dependent manner with complete inhibition at $20 \mu \mathrm{M}$ (Figure 3A). However, the levels of cyclin D1, CDK4 and CDK6 proteins were not affected by baicalin treatment. Cyclin $\mathrm{E}$ is known to play a role in the $\mathrm{G} 0 / \mathrm{G} 1$ transition. Coinciding with the onset of cell cycle arrest, the increased cyclin E level after 24-48 h of PDGF-BB stimulation was reduced in baicalin-pretreated VSMCs (Figure 3B). Cyclins and CDKs regulate phosphorylation of several substrates, including p27. Specifically, the active cyclin E-CDK2 complex has been shown to regulate p27 protein level through phosphorylation on Thr187 [14]. This phosphorylation is a prerequisite for the proteasomedependent degradation of p27 [15]. Therefore, the observed baicalin-induced increase in p27 protein levels may have resulted from the downregulation of cyclin E-CDK2 activation. To confirm this, we performed coimmunoprecipitation using anti-cyclin $\mathrm{E}$ and anti-CDK2 antibodies. Cyclin E-CDK2 complex formation induced by PDGF-BB was concentration-dependently reduced by pretreatment of baicalin in VSMCs (Figure 3C). To address whether there is a causal relationship between the reduction of cyclin E-CDK2 complexes and the upregulation of p27, the phosphorylation of p27 was detected 
A

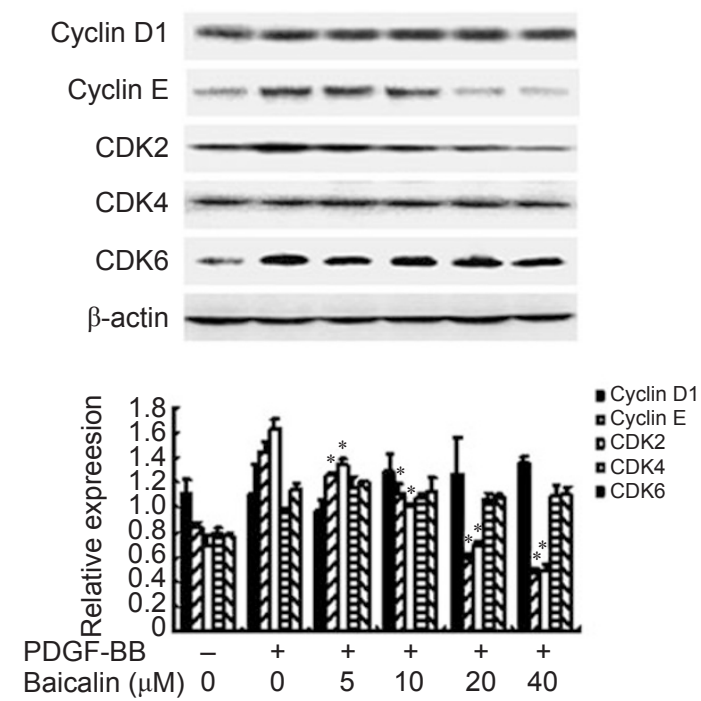

B

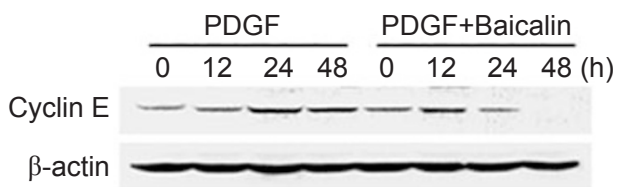

C

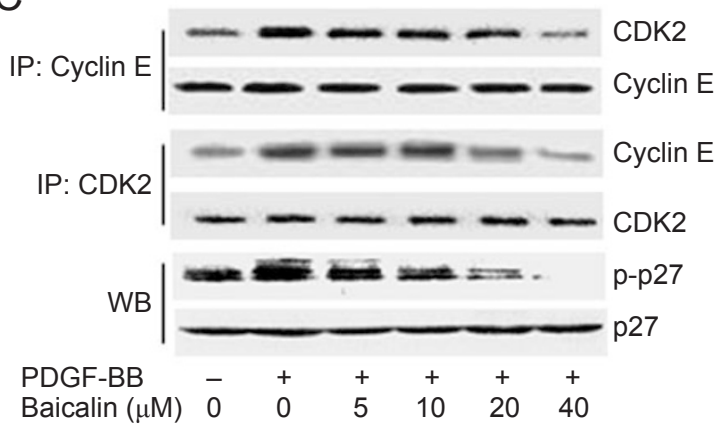

D
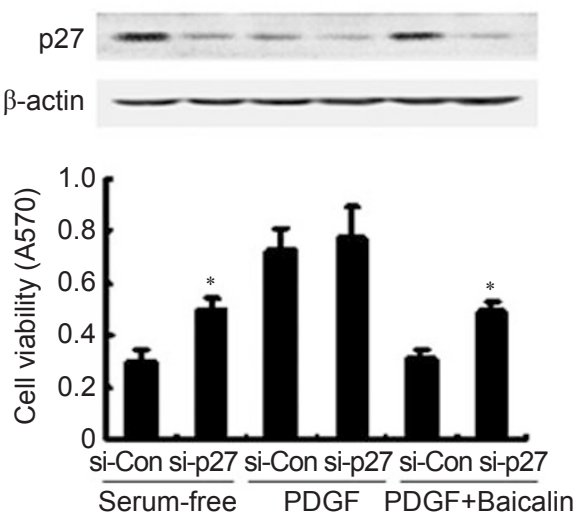

Figure 3 Baicalin inhibits expression and activation of cyclin E and CDK2 in VSMCs. (A) Western blotting. VSMCs were pretreated by baicalin at indicated concentration for $24 \mathrm{~h}$, and then stimulated with PDGF-BB for additional $24 \mathrm{~h}$. $\beta$-Actin was used as an internal control. The graphs represent the relative level of these proteins for four independent experiments. $* P<0.05$, compared with PDGF-BB. (B) Western blotting. VSMCs were stimulated with PDGF-BB for indicated time periods with or without baicalin $(20 \mu \mathrm{M})$ pretreatment. (C) Cross co-immunoprecipitation (IP) and western blotting (WB). VSMCs were stimulated with PDGF-BB for $3 \mathrm{~h}$ following baicalin pretreatment. (D) Western blotting (upper) and MTT assay (below). VSMCs were transfected with siRNA for $20 \mathrm{~h}$, and then stimulated with PDGF-BB for $24 \mathrm{~h}$ with or without baicalin pretreatment. Serum-free was used as positive control. $* P<0.05$, compared with si-Control.

using an anti-phospho-p27 antibody under the same conditions. As expected, the reduction of phosphorylated p27, as a result of baicalin treatment, was linked to the downregulation of cyclin E-CDK2 complex formation (Figure 3C). Thus, baicalin may increase p27 protein stability by inhibiting its phosphorylation. To test the involvement of p27 in baicalin-induced growth arrest, we examined the effects of p27 knockdown by specific small interfering RNA (siRNA). Our results showed that p27 knockdown impaired the growth suppression induced by baicalin (Figure 3D). Taken together, pretreatment of baicalin inhibits cyclin E-CDK2 activation and upregulates p27 protein levels in PDGF-BB-stimulated VSMCs.

Baicalin inhibits PDGFR $\beta$-ERK signaling cascade activated by PDGF-BB in VSMCS

To further delineate the cellular and molecular mechanisms underlying baicalin-induced VSMC growth inhibi- tion, we evaluated the effect of baicalin on the mitogenactivated protein kinase (MAPK) signaling cascades. After pretreatment with baicalin, VSMCs were stimulated with PDGF-BB for $30 \mathrm{~min}$, and the phosphorylation status of ERK, c-Jun NH2-terminal kinase (JNK), p38 MAPK, and Akt was measured by western blot analysis using antibodies that identify the active (phosphorylated) forms of these kinases. Baicalin markedly inhibited MEK-ERK1/2 activation by PDGF-BB in a concentration-dependent manner in VSMCs, which was associated with a reduction of PDGFR $\beta$ phosphorylation, while there were no changes in the phosphorylated forms of JNK, p38 and Akt (Figure 4A). To elucidate the mechanism by which baicalin inhibits PDGFR $\beta$ phosphorylation induced by PDGF-BB, cells were treated simultaneously with both PDGF-BB $(10 \mathrm{ng} / \mathrm{ml})$ and baicalin $(20 \mu \mathrm{M})$ or with a pre-mixture of both at the same concentrations. Under such conditions, no changes 
were observed in PDGFR $\beta$ phosphorylation compared to PDGF-BB-stimulated cells (Figure 4B). We thus speculate that baicalin pretreatment may result in the impaired response capacity of PDGFR $\beta$ to stimuli. To confirm this possibility, the phosphorylation of ERK1/2 and p27, as well as cyclin E-CDK2 complex formation was exam-
A

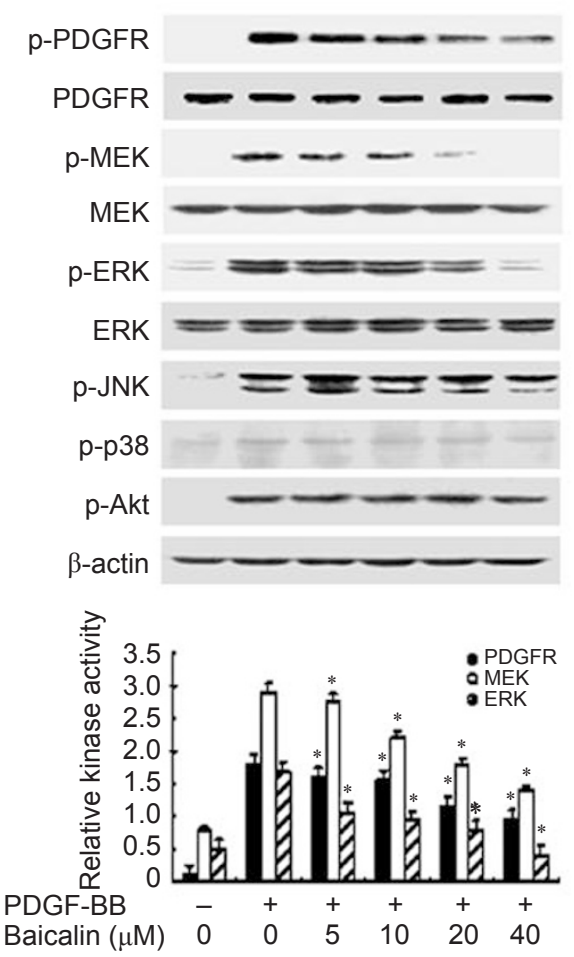

C

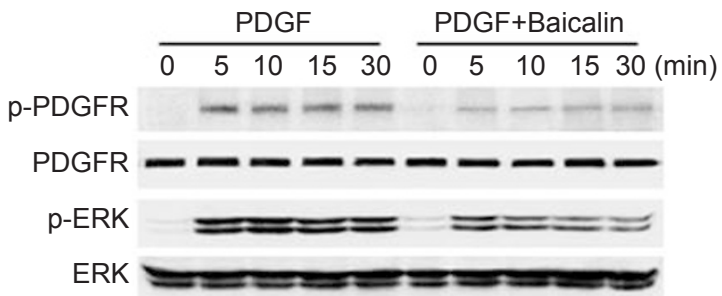

B
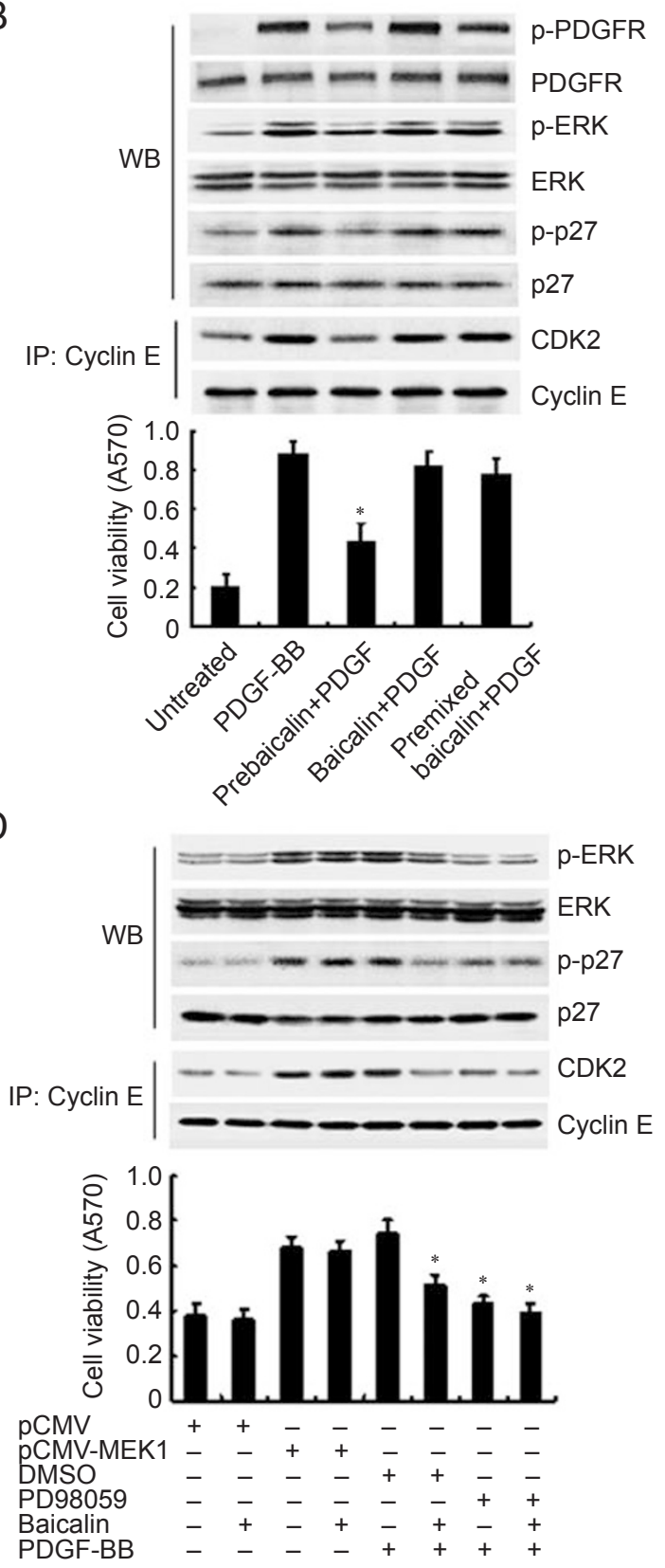

Figure 4 Baicalin inhibits PDGFR $\beta$-ERK1/2 signaling cascade activated by PDGF-BB in VSMCs. (A) Western blotting. VSMCs were stimulated with PDGF-BB for $30 \mathrm{~min}$ following pretreatment with baicalin at the indicated concentration. $\beta$-Actin was used as an internal control. The graphs represent the relative activity of these kinases for four independent experiments. $* P<0.05$, compared with PDGF-BB. (B) Western blotting (WB), co-immunoprecipitation (IP) and MTT assay. VSMCs were stimulated with PDGF-BB for 30 min (for PDGFR and ERK1/2), $3 \mathrm{~h}$ (for p27 and IP), and $24 \mathrm{~h}$ (for cell viability) with treatment by baicalin in indicated manners. ${ }^{*} P<0.05$, compared with PDGF-BB. (C) Western blotting. VSMCs were stimulated with PDGF-BB for indicated time periods with or without pretreatment by baicalin $(20 \mu \mathrm{M})$. (D) Western blotting (WB), co-immunoprecipitation (IP), and MTT assay. VSMCs were stimulated with PDGF-BB as in B following treatment by PD98059 $(20 \mu \mathrm{M})$ or transfection by pCMV-MEK1 for $24 \mathrm{~h}$, with or without baicalin pretreatment. $* P<0.05$, compared with PDGF-BB. 
ined under the same experimental conditions. The reduction of phosphorylated p27 and cyclin E-CDK2 complex formation was only found in baicalin-pretreated VSMCs following $3 \mathrm{~h}$ of PDGF-BB stimulation, paralleled with the inhibition of PDGFR $\beta$-ERK1/2 signaling cascade and cell proliferation (Figure 4B). In addition, the inhibitory effect of baicalin on ERK1/2 activation downstream of PDGFR signaling was further supported by parallel changes in phosphorylation levels of PDGFR $\beta$ and ERK1/2 after PDGF-BB stimulation with or without baicalin pretreatment. The phosphorylated forms of PDGFR $\beta$ and ERK1/2 were decreased in a time-dependent manner, starting from 5 min of PDGF-BB treatment, in baicalin-pretreated VSMCs, with no changes of the total proteins, demonstrating an actual decrease in phosphorylation levels, indicative of reduced activity (Figure 4C). Because treatment with PD98059, a specific inhibitor of ERK1/2, reduced p27 phosphorylation and cell proliferation, which was similar to the effects of baicalin (Figure 4D), the inhibition of ERK activation may be an alternative mechanism for suppression of $\mathrm{p} 27$ phosphorylation by baicalin. To test this, VSMCs were transfected with a constitutively active MEK expression plasmid (pCMV-MEK1), and then treated with baicalin and PDGF-BB. We found that increase of ERK1/2 activity impaired the inhibitory effect of baicalin on p27 phosphorylation in VSMCs, and the growth inhibition by baicalin was abolished under such conditions (Figure 4D). Further, the relationship between ERK1/2 activation and cyclin E-CDK2 complex formation was examined by coimmunoprecipitation under the same conditions. Treatment of baicalin and/or PD98059 resulted in reduction of cyclin E-CDK2 complex formation along with inhibition of ERK $1 / 2$ activation, while the opposite change was found upon ERK1/2 activation by the overexpression of a constitutively active MEK (Figure 4D). These results suggest that reduced cyclin E-CDK2 activity is likely an event downstream of the blockade of ERK1/2 pathway by baicalin.

\section{Baicalin inhibits adhesion molecule expression and} $V S M C$ migration induced by PDGF-BB

PDGF initiates a multitude of biological effects through the activation of intracellular signal transduction pathways that contribute to both VSMC proliferation and migration. We examined the effect of baicalin on PDGFBB-induced VSMC migration by the cell monolayer wounding assay that represents chemokinesis. Treatment with PDGF-BB for $12 \mathrm{~h}$ increased the basal migration of VSMCs by $\sim$ fourfold. Compared with PDGF-BB alone, baicalin pretreatment caused a $60 \%$ reduction of cell migration, but no change was found in VSMCs treated simultaneously with both PDGF-BB and baicalin or with a pre-mixture of PDGF-BB and baicalin (Figure 5A). Then, we examined the effect of baicalin on several migration regulatory proteins, including osteopontin (OPN), intercellular adhesion molecule 1 (ICAM-1), vascular cell adhesion molecule 1 (VCAM-1) and matrix metalloprotease 2 (MMP-2). Baicalin pretreatment inhibited the upregulation of OPN, ICAM-1, VCAM-1 and MMP2 proteins by PDGF-BB in a concentration-dependent manner (Figure 5B). A measure of $20 \mu \mathrm{M}$ baicalin resulted in an $\sim 40 \%$ decrease in the levels of these proteins compared to that in PDGF-BB-treated cells, and a $60 \%$
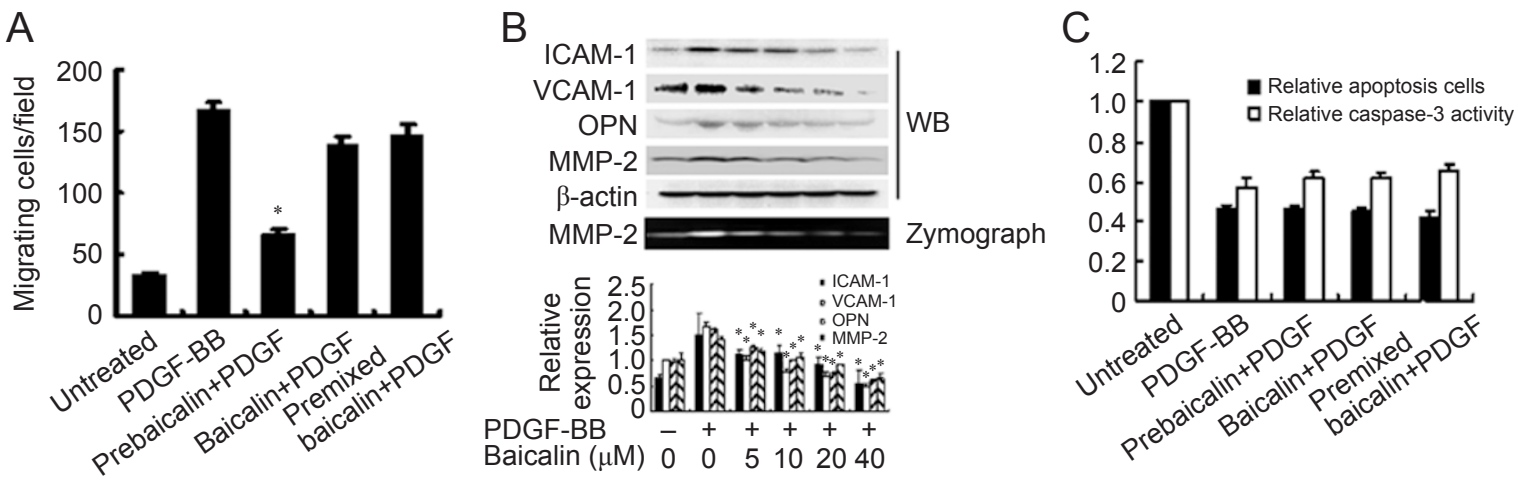

Figure 5 Baicalin inhibits adhesion molecule expression and migration induced by PDGF-BB in VSMCs. (A) Migration assay. VSMCs were stimulated with PDGF-BB for $12 \mathrm{~h}$ with or without treatment by baicalin in indicated manners. $* P<0.05$, compared with PDGF-BB. (B) Western blotting (WB) and Zymograph. $\beta$-Actin was used as an internal control. VSMCs were stimulated with PDGF-BB for $24 \mathrm{~h}$ following baicalin pretreatment at indicated concentration. The graphs represent the relative level of these proteins for four independent experiments. ${ }^{*} P<0.05$, compared with PDGF-BB. (C) Apoptosis analysis by flow cytometry (black) and caspase-3 activity assay (white). VSMCs were stimulated with PDGF-BB for $24 \mathrm{~h}$ with or without treatment by baicalin in indicated manners. 
decrease was observed with $40 \mu \mathrm{M}$ baicalin (Figure 5B). Consistent with the decrease in MMP-2 protein levels, zymography assay showed that baicalin $(20 \mu \mathrm{M})$ decreased MMP-2 activity by $70 \%$, compared with that induced by PDGF-BB alone (Figure 5B). These findings suggest that baicalin inhibits the migration of VSMCs induced by PDGF-BB via suppressing the expression of migration-related proteins in these cells. Flow cytometry analysis showed no effect of baicalin on apoptosis of PDGF-BB-stimulated VSMCs, which was verified by no change in caspase- 3 activity (Figure $5 \mathrm{C}$ ).

\section{Baicalin inhibits neointimal hyperplasia}

To investigate the role of baicalin in regulating VSMC proliferation in vivo, rat carotid arteries were harvested on day 28 after balloon injury, and an increased intimato-media (I/M) thickness ratio of carotid arteries was observed (Figure 6A). Administration with baicalin (70 $\mathrm{mg} / \mathrm{kg} /$ day) significantly reduced $\mathrm{I} / \mathrm{M}$ thickness ratio by over 55\% compared with injured controls (Figure $6 \mathrm{~A}$ ), and inhibited the injury-induced increase in PCNA expression in neointima of carotid arteries, which was accompanied with an increase in $\mathrm{p} 27$ expression and attenuation of neointimal hyperplasia (Figure 6B). However, lower doses of baicalin $(\leq 35 \mathrm{mg} / \mathrm{kg} /$ day $)$ showed only a slight inhibitory effect on neointimal hyperplasia, which did not reach statistic significance (data not shown). These results suggest that baicalin exhibits an inhibitory effect on cell proliferation in vivo, and may be an effective agent for prevention of restenosis after angioplasty.

\section{Discussion}

PDGF-BB plays an important role in vascular remodeling during cellular and extracellular responses to injury $[16,17]$. It has been suggested that different signaling pathways mediated by PDGFR $\beta$ regulate the proliferative and the migratory responses to PDGF-BB, with MAPK regulating proliferation and Akt regulating migration [18]. In this study, we show that baicalin has potent and concentration-dependent inhibitory effects on PDGFBB-stimulated VSMC proliferation and migration without inducing apoptosis (IC50 of $16.39 \mu \mathrm{M}$ for the induction of G0-G1-phase arrest), and that it does not affect the viability of quiescent VSMCs. Baicalin pretreatment inhibits PDGFR $\beta$ phosphorylation in a concentrationdependent manner and this inhibitory effect is associated with the inhibition of downstream ERK1/2 signaling events triggered by the receptor, including the reduction of 27 phosphorylation and cyclin E-CDK2 complex formation. As treatment with the specific ERK1/2 inhibitor, PD98059, reveals a similar effect as baicalin, the inhibi-
A

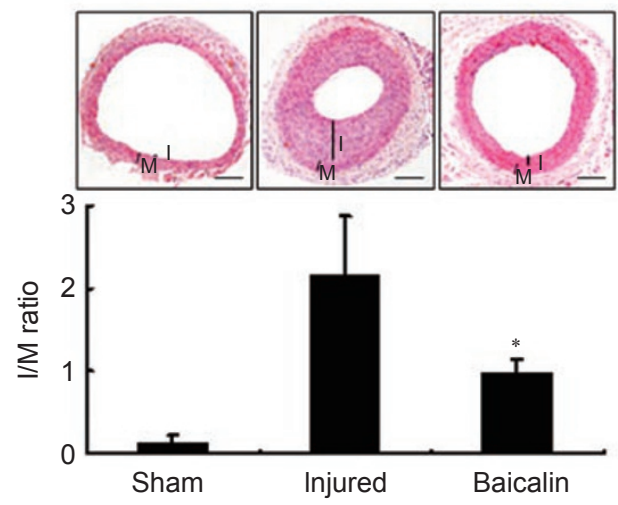

B
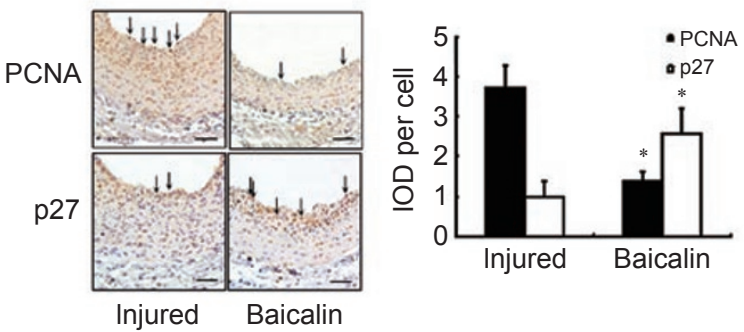

Figure 6 Baicalin inhibits neointimal hyperplasia. The sections of rat carotid arteries were prepared on day 28 after balloon injury. (A) $\mathrm{H}$ and $\mathrm{E}$ staining (upper) and I/M thickness ratio analysis (below). The bar indicates $200 \mu \mathrm{m}$. $* P<0.05$, compared with injured group $(n=6)$. (B) Immunohistochemistry analysis. The graphs represent the integrated optical density per cell of intima. Representative positive signals (brown) are indicated by arrows. The bar indicates $50 \mu \mathrm{m}$. $* P<0.05$, compared with injured group $(n=6)$.

tion of ERK1/2 activation is likely a major mechanism of growth suppression by baicalin. Nevertheless, the inhibition of tyrosine phosphorylation of PDGFR $\beta$ by baicalin did not occur when VSMCs were treated simultaneously with both PDGF-BB and baicalin or with a pre-mixture of the two agents, suggesting that pretreatment of baicalin likely acts by impairing the response capacity of PDGFR $\beta$ to PDGF-BB stimulation. Baicalin belongs to a class of polyphenols. Previous studies have shown that polyphenols inhibit PDGFR $\beta$ autophosphorylation [1922]. Although the mechanisms underlying the inhibitory effect of these polyphenols remain to be established, previous studies suggested that some of these molecules may inhibit the activation of receptor tyrosine kinase by competing with adenosine triphosphate for binding to the kinase domain of the receptor, resulting in impaired activation of key signaling intermediates [23, 24]. We speculate that such a mechanism could also be involved in the inhibitory effect of baicalin toward PDGFR $\beta$. In 
addition, the involvement of ERK1/2 was further supported by the fact that transient expression of a constitutively active mutant of MEK abolished the inhibitory effect of baicalin on cell proliferation, which was accompanied with an increase in cyclin E-CDK2 activation and reduction of p27 level. However, baicalin failed to attenuate PDGF-stimulated phosphorylation of JNK, p38 MAPK, and Akt. Our results suggest that blockade of the PDGFR $\beta$-ERK $1 / 2$ pathway is involved in baicalin-induced proliferation suppression in PDGF-BB-stimulated VSMCs. It is currently unknown how baicalin enters the cell and/or reacts with the compartment where the kinases are localized. Also, baicalin may have no effect on kinase activities in quiescent cells and only interfere with the ATP-binding site when the enzyme translocates upon activation [25].

Using RNAi-mediated knockdown, we have found that the increase in p27 accumulation is involved in suppression of proliferation induced by baicalin in VSMCs. Recent studies have suggested a physiological role of p27 as a regulator of VSMC growth during the pathogenesis of cardiovascular diseases [26]. We focused on the regulatory mechanism of $\mathrm{p} 27$ induction by baicalin and found that baicalin-induced upregulation of $\mathrm{p} 27$ is associated not only with the blockade of PDGFR $\beta$-ERK $1 / 2$ signaling but also with the reduction of cyclin E-CDK2 activation in VSMCs. Treatment of baicalin and/or PD98059 results in reduction of p27 phosphorylation along with inhibition of ERK1/2 activation, while the opposite change is found upon ERK1/2 activation by the overexpression of a constitutively active MEK. In addition, we show that the expression and activation of CDK2 and cyclin E markedly decrease in baicalin-pretreated VSMCs, which is associated with p27 accumulation and cell cycle arrest. The reduction of phosphorylated p27 as a result of baicalin treatment is linked to the downregulation of cyclin E-CDK2 complex formation. Cyclin E-CDK2 may phosphorylate p27 and result in its elimination [27]. It is now clear that the level of p27 protein is mainly controlled by its rate of degradation rather than by changes in transcription or translation [28]. Thus, two possibilities could account for the inhibition of $\mathrm{p} 27$ phosphorylation by baicalin observed in this study. Suppression of ERK1/2 activation may directly result in reduced p27 phosphorylation, and/or do so indirectly via inhibiting cyclin E-CDK2 activation. Previous study showed that baicalin had antiproliferation effects on hepatocytes via TGF- $\beta 1$ secretion from Kupffer cells, which was related to the activation of NF- $\kappa B$ and PKC pathways [29]. In cancer cell lines, baicalin induced apoptosis and proliferation retardation, which may be related to the activation of p38 MAPK and Akt [8]. Our data indicate that baicalin may increase $\mathrm{p} 27$ protein stability by inhibiting its phosphorylation and degradation, which is required for baicalin-induced growth arrest in VSMCs.

There is much evidence that enhanced proliferation and migration of VSMCs is a fundamental feature of the pathogenesis of atherosclerosis and other vascular proliferative disorders. Thus, prevention of pathological VSMC proliferation still remains as a major clinical challenge, which underscores the need for new therapeutic strategies. In the present study, we have also shown using an animal arterial balloon-injury model characterized by PDGF receptor upregulation [16] that baicalin $(70 \mathrm{mg} / \mathrm{kg} /$ day) significantly inhibits neointimal formation, accompanied with reduction of cell proliferation after vascular injury in rats. These results substantiate our in vitro findings and strongly support the notion that baicalin confers protection against injury-induced pathological vascular remodeling. Lower dose of baicalin ( $\leq$ $35 \mathrm{mg} / \mathrm{kg} /$ day), however, did not significantly inhibit the injury-induced neointima formation. Pharmacokinetics study showed that the major forms in the serum are their conjugated metabolites after oral administration of the polyphenols, including baicalin and baicalein, indicating a high level of biotransformation [30]. Moreover, the bioavailability of baicalin is relatively low among polyphenols. Our findings point to the possibility that lower inhibitory effect of baicalin on cell proliferation in vivo may result from its high level of biotransformation and/ or low bioavailability. Because the principle metabolites in vivo possess completely different physicochemical properties from the free form [30], it is hard to directly infer the in vivo effects of this compound from its in vitro activities. The bioavailability and bioactivity of baicalin would be an important issue to consider for any future clinical applications.

In summary, baicalin induces growth arrest in PDGFBB-stimulated VSMCs, and prevents neointimal hyperplasia after balloon injury. This process is associated with inhibition of cyclin E-CDK2 activation, and subsequent increase in p27 accumulation via blockade of the PDGFR $\beta$-ERK1/2 signaling cascade.

\section{Materials and Methods}

\section{Reagents}

Recombinant human PDGF-BB was purchased from R\&D Systems Inc. Baicalin and baicalein was purchased from Sigma Co and dissolved in DMSO, and the concentration of DMSO was < $0.8 \%$ in the control and drug-containing medium.

\section{VSMC culture and treatment}

VSMCs were obtained from thoracic aorta of male SpragueDawley rats weighing between 100 and $200 \mathrm{~g}$, as described [7]. All 
of the experiments were performed using 3-5 passages of VSMCs. Before isolating, VSMCs were pretreated by different concentrations of baicalin for $24 \mathrm{~h}$ and then stimulated with PDGF-BB (10 $\mathrm{ng} / \mathrm{ml}$ ) for indicated time periods. This study was performed via a protocol approved by the Institutional Animal Care and Use Committee of Hebei Medical University in accordance with the Guide for the Care and Use of Laboratory Animals.

\section{Cell proliferation assay}

VSMCs were seeded onto 96 -well plates $\left(2 \times 10^{3}\right.$ cells per well) and pretreated with various concentrations of baicalin or baicalein for $24 \mathrm{~h}$ before stimulation with or without PDGF-BB (10 $\mathrm{ng} / \mathrm{ml}$ ). Proliferation was measured using MTT assay [31].

\section{Fluorescence-activated cell sorting analysis}

VSMCs were collected after $24 \mathrm{~h}$ of treatment with or without of PDGF-BB $(10 \mathrm{ng} / \mathrm{ml})$ and various concentrations of baicalin, fixed in $70 \%$ ethanol, washed twice with PBS, and stained with a $50 \mu \mathrm{g} / \mathrm{ml}$ propidium iodide solution containing $0.1 \%$ Triton $\mathrm{X}-100$, $0.1 \mathrm{mM}$ EDTA, and $50 \mu \mathrm{g} / \mathrm{ml}$ RNase A. Fluorescence was measured and analyzed using a FACSCalibur flow cytometer (Becton Dickinson Immunocytometry Systems).

\section{Cell migration assay}

The cell migration was measured using a monolayer wounding protocol in which cells migrated from a confluent area into an area that was mechanically denuded of cells [31]. Three different fields of migration were photographed with a video camera system using Scion Image software at the intersection of the previously marked line and wound edge before and after treatment with PDGF-BB $(10 \mathrm{ng} / \mathrm{ml})$ for $12 \mathrm{~h}$ in the presence or absence of baicalin $(20 \mu \mathrm{M})$. The migration activity was expressed as the number of cells migrating into the wound in each field.

\section{Cell transfection}

The constitutively active ERK kinase MEK1 plasmid (pCMVMEK1) was kindly provided by Kun-Liang Guan (Moore's Cancer Center, CA, USA). VSMCs were transfected with pCMV and pCMV-MEK1 using Lipofectamine 2000 (Invitrogen) according to the manufacturer's instructions. After transfection for $24 \mathrm{~h}$, VSMCs were stimulated with PDGF-BB and then treated with baicalin $(20 \mu \mathrm{M})$ for $24 \mathrm{~h}$.

\section{siRNA transfection}

The siRNA duplex targeting rat p27 mRNA (5'-UUG CCG AGA UAU GGA AGA ATT-3') was synthesized from TaKaRa. Scrambled siRNA (si-Control) served as a negative control. The siRNA was transiently transfected into VSMCs using Lipofectamine 2000 reagent (Invitrogen) according to the manufacturer's protocol. At $20 \mathrm{~h}$ after transfection, the VSMCs were treated with baicalin and PDGF-BB as above mentioned.

\section{Western blot analysis}

Lysates from VSMCs were prepared as previously described [6]. Equal amounts of protein $(60-100 \mu \mathrm{g})$ were separated by $10 \%$ SDS-PAGE and electrotransferred to a PVDF membrane. Membranes were blocked, and then incubated with anti-cyclin D1 (1:300), anti-cyclin E (1:400), anti-CDK2 (1:300), anti-CDK4 (1:300), anti-CDK6 (1:300), anti-PCNA (1:300), anti-p21 (1:300), anti-p27 (1:300), anti-phospho-p27 (1:300), anti-phospho-JNK (Tyr183/185; 1:300), anti-p38 MAPK (1:300), anti-phospho-p38 MAPK (Thr180/Tyr182; 1:300), anti-MEK1/2 (1:300), antiphospho-MEK1/2 (Ser217/221; 1:400), anti-ERK1/2 (1:300), antiphospho-ERK1/2 (Tyr204; 1:300), anti-phospho-Akt (1:300), anti-PDGFR $\beta$, anti-phospho-PDGFR $\beta$ (Tyr751; 1:300), anti-OPN (1:300), anti-ICAM-1 (1:300), anti-VCAM-1 (1:300), anti-MMP-2 $(1: 300)$, and anti- $\beta$-actin (1:300) antibodies (Santa Cruz Biotechnology) overnight, and then with the horseradish peroxidaseconjugated secondary antibody (Santa Cruz Biotechnology) (1:10 $000)$ for $2 \mathrm{~h}$. The blot was detected with the enhanced chemiluminescence detection system (Santa Cruz Biotechnology). The experiments were replicated four times.

\section{Co-immunoprecipitation assay}

VSMCs were harvested after PDGF-BB treatment for $3 \mathrm{~h}$ with or without baicalin pretreatment. Cells were lysed in a buffer composed of $50 \mathrm{mM}$ Tris- $\mathrm{HCl}(\mathrm{pH} 7.6), 150 \mathrm{mM} \mathrm{NaCl}, 1 \% \mathrm{NP}-40,10$ $\mathrm{mM}$ sodium phosphate, $10 \mathrm{mM} \mathrm{NaF}, 1 \mathrm{mM}$ sodium orthovanadate, $2 \mathrm{mM}$ phenylmethylsulfonyl fluoride, $10 \mu \mathrm{g} / \mathrm{ml}$ aprotinin, $10 \mu \mathrm{g} /$ $\mathrm{ml}$ leupeptin, and $10 \mu \mathrm{g} / \mathrm{ml}$ pepstatin. After centrifugation, $50 \mu \mathrm{g}$ of the clarified cell lysate was incubated with $15 \mu$ l of protein $G$ plus/protein A-agarose (Santa Cruz Biotechnology) and $1 \mu \mathrm{g}$ of anti-CDK2, anti-cyclin E antibodies. After $24 \mathrm{~h}$ incubation, the immune complexes were centrifuged and washed four times with ice-cold lysis buffer. The immunoprecipitated protein was further analyzed by western blot as described above.

\section{Caspase activity assays}

Caspase- 3 activity was analyzed according to the manufacturer's instructions with the caspase colorimetric assay kit (Promega Co). Cells were harvested, lysed in lysis buffer for $30 \mathrm{~min}$, and vibrated for $10 \mathrm{~s}$ at $4{ }^{\circ} \mathrm{C}$. After centrifugation, the supernatant was collected and the protein concentration was determined. Each sample $(50 \mu \mathrm{l})$ was incubated with caspase- 3 substrate $(5 \mu \mathrm{l})$ and $2 \times$ reaction buffer $(50 \mu \mathrm{l})$ at $37^{\circ} \mathrm{C}$ for $4 \mathrm{~h}$ and measured by chromatography at $405 \mathrm{~nm}$ wavelength.

\section{Balloon injury and morphometric analysis of neointima for- mation}

Balloon denudation of the left common carotid artery of male Sprague-Dawley rats was performed, as described [32]. Baicalin was freshly dissolved in 10\% polyglycol-400 and orally administered at doses of $17.5,35$, and $70 \mathrm{mg} / \mathrm{kg} /$ day by gastric gavage from 3 days before balloon injury to 28 days after the injury $(n=$ 6 per group). The arteries were collected at day 28 after balloon injury, and embedded in paraffin to prepare cross sections. Neointima thickening was assessed using the I/M thickness ratio measured from haematoxylin- and eosin-stained arterial cross sections with a computer-based Image-Pro Morphometric System in a double-blind manner. Four discontinuous sections from each vessel were measured in a Sprague-Dawley rat, whereas six rats were used in each experimental group.

\section{Immunohistochemistry analysis}

Standard immunohistochemical techniques were used with antiPCNA (1:100) and anti-p27 (1:100) antibodies and a biotinylated anti-mouse secondary antibody (1:100) on perfusion-fixed, paraffin-embedded tissues. Slides were treated with avidin-biotin block 
and exposed to diaminobenzidine black chromogen with nuclear fast red counterstain. Slides were coverslipped for analysis under light microscopy. Staining intensities were determined by measurement of the integrated optical density with light microscopy using a computer-based Image-Pro Morphometric System by two independent observers in a double-blind manner.

\section{Zymography}

MMP-2 activity in the medium was analyzed by non-reducing SDS-PAGE in $10 \%$ gels containing $0.1 \%$ gelatin [33]. Samples were denatured at room temperature in an equal volume of 0.25 M Tris- $\mathrm{HCl}$ (pH 6.8), 20\% glycerol, 2\% SDS, and $10 \mu \mathrm{g} / \mathrm{ml}$ bromophenol blue. After electrophoresis, MMP-2 was renatured by rinsing the gel in $2.5 \%$ Triton $\mathrm{X}-100$ at room temperature for 30 $\mathrm{min}$, and then activated in reaction buffer ( $50 \mathrm{mM}$ Tris- $\mathrm{HCl}(\mathrm{pH} 7.6)$ containing $0.2 \mathrm{M} \mathrm{NaCl}, 5 \mathrm{mM} \mathrm{CaCl}_{2}$, and $0.02 \%$ Brij 35) at $37{ }^{\circ} \mathrm{C}$ for $18 \mathrm{~h}$. The gels were stained for $90 \mathrm{~min}$ with $0.5 \%$ Coomassie brilliant blue R250 and destained with $10 \%$ acetic acid in $40 \%$ methanol. MMP-2 activity was evident as a clear band against the blue background of stained gelatin.

\section{Data statistics}

Data are presented as means \pm SEM. ANOVA and paired or unpaired $t$-test were performed for statistical analysis as appropriate. $P<0.05$ was considered statistically significant.

\section{Acknowledgments}

We are grateful to Dr Guan KL (Moore's Cancer Center, La Jolla, CA, USA) for the gift of pCMV-MEKca. This study was supported by the National Natural Science Foundation of China (30770787 and 90919035), the National Basic Research Program of China (2005CB523301), and the International Cooperation in Science and Technology Projects (2006DFB32460) and the Hebei Province Natural Science Foundation (C2007000831).

\section{References}

1 Dzau VJ, Braun-Dullaeus RC, Sedding DG. Vascular proliferation and atherosclerosis: new perspectives and therapeutic strategies. Nat Med 2002; 8:1249-1256.

2 Huang M, Duhadaway JB, Prendergast GC, Laury-Kleintop LD. RhoB regulates PDGFR-beta trafficking and signaling in vascular smooth muscle cells. Arterioscler Thromb Vasc Biol 2007; 27:2597-2605.

3 Sherr CJ. Cancer cell cycles. Science 1996; 274:1672-1677.

4 Egozi D, Shapira M, Paor G, Ben-Izhak O, Skorecki K, Hershko DD. Regulation of the cell cycle inhibitor p27 and its ubiquitin ligase Skp2 in differentiation of human embryonic stem cells. FASEB J 2007; 21:2807-2817.

5 Kossatz U, Malek NP. p27: tumor suppressor and oncogene ...? Cell Res 2007; 17:832-833.

$6 \mathrm{Hu}$ Y, Cheng L, Hochleitner BW, Xu Q. Activation of mitogen-activated protein kinases (ERK/JNK) and AP-1 transcription factor in rat carotid arteries after balloon injury. Arterioscler Thromb Vasc Biol 1997; 17:2808-2816.

7 Zheng B, Han M, Bernier M, et al. Krüppel-like factor 4 inhibits proliferation by platelet-derived growth factor receptor $\beta$-mediated, not by retinoic acid receptor $\alpha$-mediated, phosphatidylinositol 3-kinase and ERK signaling in vascular smooth muscle cells. J Biol Chem 2009; 284:22773-22785.

8 Chao JI, Su WC, Liu HF. Baicalein induces cancer cell death and proliferation retardation by the inhibition of CDC2 kinase and survivin associated with opposite role of p38 mitogenactivated protein kinase and AKT. Mol Cancer Ther 2007; 6:3039-3048.

9 Ma Z, Otsuyama K, Liu S, et al. Baicalein, a component of Scutellaria radix from Huang-Lian-Jie-Du-Tang (HLJDT), leads to suppression of proliferation and induction of apoptosis in human myeloma cells. Blood 2005; 105:3312-3318.

10 Lee HZ, Leung HW, Lai MY, Wu CH. Baicalein induced cell cycle arrest and apoptosis in human lung squamous carcinoma CH27 cells. Anticancer Res 2005; 25:959-964.

11 Woo AY, Cheng CH, Waye MM. Baicalein protects rat cardiomyocytes from hypoxia/reoxygenation damage via a prooxidant mechanism. Cardiovasc Res 2005; 65:244-253.

12 Chang CP, Huang WT, Cheng BC, Hsu CC, Lin MT. The flavonoid baicalin protects against cerebrovascular dysfunction and brain inflammation in experimental heatstroke. Neuropharmacology 2007; 52:1024-1033.

13 Toda N, Ayajiki K, Okamura T. Cerebral blood flow regulation by nitric oxide: recent advances. Pharmacol Rev 2009; 61:62-97.

14 Sheaff RJ, Groudine M, Gordon M, Roberts JM, Clurman BE. Cyclin E-CDK2 is a regulator of p27Kip1. Genes Dev 1997; 11:1464-1478.

15 Vlach J, Hennecke S, Amati B. Phosphorylation-dependent degradation of the cyclin-dependent kinase inhibitor p27. EMBO J 1997; 16:5334-5344.

16 Majesky MW, Reidy MA, Bowen-Pope DF, Hart CE, Wilcox JN, Schwartz SM. PDGF ligand and receptor gene expression during repair of arterial injury. J Cell Biol 1990; 111:21492158.

17 Heldin $\mathrm{CH}$, Westermark B. Mechanism of action and in vivo role of platelet-derived growth factor. Physiol Rev 1999; 79:1283-1316.

18 Yang X, Thomas DP, Zhang X, et al. Curcumin inhibits platelet-derived growth factor-stimulated vascular smooth muscle cell function and injury-induced neointima formation. Arterioscler Thromb Vasc Biol 2006; 26:85-90.

19 Lamy S, Beaulieu E, Labbe D, et al. Delphinidin, a dietary anthocyanidin, inhibits platelet-derived growth factor ligand/ receptor (PDGF/PDGFR) signaling. Carcinogenesis 2008; 29:1033-1041.

20 Godichaud S, Si-Tayeb K, Auge N, et al. The grape-derived polyphenol resveratrol differentially affects epidermal and platelet-derived growth factor signaling in human liver myofibroblasts. Int J Biochem Cell Biol 2006; 38:629-637.

21 Chen A, Zhang L. The antioxidant (-)-epigallocatechin-3gallate inhibits rat hepatic stellate cell proliferation in vitro by blocking the tyrosine phosphorylation and reducing the gene expression of platelet-derived growth factor-beta receptor. $J$ Biol Chem 2003; 278:23381-23389.

22 Ahn HY, Hadizadeh KR, Seul C, Yun YP, Vetter H, Sachinidis A. Epigallocathechin-3 gallate selectively inhibits the PDGFBB-induced intracellular signaling transduction pathway in vascular smooth muscle cells and inhibits transformation of sis-transfected NIH 3 T3 fibroblasts and human glioblastoma 
cells (A172). Mol Biol Cell 1999; 10:1093-1104.

23 Li M, He Z, Ermakova S, et al. Direct inhibition of insulinlike growth factor-I receptor kinase activity by (-)-epigallocatechin-3-gallate regulates cell transformation. Cancer Epidemiol Biomarkers Prev 2007; 16:598-605.

24 Yang EB, Wei L, Zhang K, Chen YZ, Chen WN. Tannic acid, a potent inhibitor of epidermal growth factor receptor tyrosine kinase. J Biochem 2006; 139:495-502.

25 Middleton E Jr., Kandaswami C, Theoharides TC. The effects of plant flavonoids on mammalian cells: implications for inflammation, heart disease, and cancer. Pharmacol Rev 2000; 52:673-751.

26 Sanz-Gonzalez SM, Poch E, Perez-Roger I, Diez-Juan A, Ivorra C, Andres V. Control of vascular smooth muscle cell growth by cyclin-dependent kinase inhibitory proteins and its implication in cardiovascular disease. Front Biosci 2000; 5:D619-628.

27 Swanson C, Ross J, Jackson PK. Nuclear accumulation of cyclin E/Cdk2 triggers a concentration-dependent switch for the destruction of p27Xic1. Proc Natl Acad Sci USA 2000; 97:7796-7801.

28 Hengst L, Reed SI. Translational control of p27Kip1 accumu- lation during the cell cycle. Science 1996; 271:1861-1864.

29 Wang JY, Chuang HN, Chiu JH, et al. Effects of Scutellaria baicalensis Georgi on macrophage-hepatocyte interaction through cytokines related to growth control of murine hepatocytes. Exp Biol Med (Maywood) 2006; 231:444-455.

30 Shia CS, Hou YC, Juang SH, et al. Metabolism and pharmacokinetics of San-Huang-Xie-Xin-Tang, a polyphenol-rich chinese medicine formula, in rats and ex-vivo antioxidant activity. Evid Based Complement Alternat Med 2009; 10:1093.

31 Li JJ, Han M, Wen JK, Li AY. Osteopontin stimulates vascular smooth muscle cell migration by inducing FAK phosphorylation and ILK dephosphorylation. Biochem Biophys Res Commun 2007; 356:13-19.

32 Andres V, Urena J, Poch E, Chen D, Goukassian D. Role of $\mathrm{Sp} 1$ in the induction of p27 gene expression in vascular smooth muscle cells in vitro and after balloon angioplasty. Arterioscler Thromb Vasc Biol 2001; 21:342-347.

33 Han M, Wen JK, Zheng B, Cheng Y, Zhang C. Serum deprivation results in redifferentiation of human umbilical vascular smooth muscle cells. Am J Physiol Cell Physiol 2006; 291:C50-C58. 